\title{
Profil de résistance aux bêta-lactamines des entérobactéries isolées des prélèvements urinaires à l'Hôpital du Mali.
}

\section{Antibiotic resistance profile of enterobacteria isolated from urinary samples at Hospital Mali.}

Kalambry AC ${ }^{1}$, Gaudré $\mathrm{N}^{2}$, Dramé BSI ${ }^{1}$, Poudiougo $\mathrm{A}^{3}$, Kassogué $\mathrm{A}^{1}$, Koné $\mathrm{H}^{1}$, Diarra $\mathrm{A}^{1}$.

1. Laboratoire d'analyse de biologie médicale de l'Hôpital du Mali, Bamako, Mali

2. Service de Médecine Vasculaire, CHU Toulouse Rangueil, Toulouse, France

3. Laboratoire d'analyse du CHU Gabriel TOURE, Bamako, Mali

Auteur Correspondant : Mr. Aimé Césaire KALAMBRY, Email : akalambry@yahoo.fr

\section{Résumé}

Le but de cette étude était de déterminer le profil bactériologique et de résistance aux antibiotiques des entérobactéries isolées dans les prélèvements urinaires de l'Hôpital du Mali. Les méthodes conventionnelles d'isolement et d'identification de la bactériologie ont été utilisées. Les milieux et galeries suivants ont été utilisés pour l'identification et l'antibiogramme : Uriselect 4, urée-indole, Muëller Hinton, Api 20E, Api 20NE. Les tests de sensibilité aux antibiotiques et l'interprétation des résultats d'antibiogramme ont été effectués respectivement par la méthode de diff\$usion des disques en milieu gélosé (de Kirby-Bauer) et selon les recommandations du Comité Antibiogramme de la Société Française de Microbiologie. Le sexe ratio H/F était de 0,8. L’âge moyen était de 36 ans. 30,0 \% des patients avaient un âge supérieur à 50 ans. La prévalence des prélèvements positifs était de 18,5\%. Les patients hospitalisés représentaient $63.2 \%$ de la population et provenaient du service de médecine interne $(50,2 \%)$ et des services de chirurgie $(35,1 \%)$. Seuls $2,0 \%$ des infections urinaires ont été retrouvées dans la tranche d'âge de 0 à 10 ans. Escherichia coli était le germe le plus isolé (42,9\%) suivi de Klebsiella pneumoniae (16,3\%). $70,0 \%$ des entérobactéries identifiées étaient résistantes à l'Amoxicilline + Acide clavulanique et 12,5\% étaient productrices de BLSE. Aucune souche n'était résistante aux carbapénèmes. La prédominance des entérobactéries, un taux extrêmement élevé de résistance à l'Amoxicilline + Acide clavulanique et une prédominance des malades hospitalisés ont été constatés. La présente étude a mis en exergue l'évolution croissante des résistances bactériennes aux antibiotiques, qui nécessite des mesures adéquates.

Mots-clés : Prélèvement urinaire, Entérobactéries ; Bêta-lactamines ; Résistance ; Hôpital du Mali

\begin{abstract}
The purpose of this study was to determine the bacteriological and antibiotic resistance profile of enterobacteria isolated in urinary tract infections at the Mali Hospital. Conventional methods of isolation and identification of bacteriology have been used. The following media and galleries were used for identification and susceptibility testing: Uriselect 4, urea-indole, Mueller Hinton, Api 20E, Api 20NE. The antibiotic susceptibility tests and the interpretation were carried out respectively by Kirby-Bauer's method (diffusion of the disks in agar medium) according to the recommendations of the antibiotic sensitivity test committee of the French Society of Microbiology. The sex-ratio $\mathrm{H} / \mathrm{F}$ was 0.8 . The average age was 36 years old. $30.0 \%$ of patients were older than 50 years. The prevalence of positive samples was $18.5 \%$. Inpatients accounted for $63.2 \%$ and came from internal medicine $(50.2 \%)$ and surgery $(35.1 \%)$. Only $2.0 \%$ of urinary tract infections were found in the age group from 0 to 10 years. Escherichia coli was the most isolated organism (42.9\%) followed by Klebsiella pneumoniae (16.3\%). $70.0 \%$ of the Enterobacteriaceae identified were resistant to amoxicillin + clavulanic acid and $12.5 \%$ were producing ESBL. No strain were resistant to carbapenem. The prevalence of Enterobacteriaceae, an extremely high level of resistance to amoxicillin + clavulanic acid and a predominance of hospitalized patients were found. The present study has highlighted the increasing evolution of bacterial resistance to antibiotics, that requires adequate measures.
\end{abstract}

Keywords: Urinary sample; Enterobacteria; Resistance; Mali Hospital. 


\section{Introduction}

Les infections du tractus urinaire constituent l'une des infections les plus fréquentes aussi bien dans la pratique hospitalière que communautaire (1). Elles sont souvent dues à des entérobactéries, principalement Escherichia coli, Klebsiella pneumoniae et Proteus sp (2). L'émergence et la diffusion des résistances aux antibiotiques représentent une réelle menace pour la santé publique mondiale (3). Selon l'Organisation Mondiale de la Santé (OMS), l'infection urinaire constitue un véritable problème majeur de santé publique. Environ 700000 personnes dans le monde meurent chaque année en raison d'infections résistantes aux médicaments et, si aucune mesure n'est prise, on estime que ces infections tueront 10 millions de personnes par an d'ici 2050. La situation est alarmante dans les pays à ressources limitées où les maladies infectieuses, la pauvreté et la malnutrition sont endémiques. La survenue des résistances aux antibiotiques est un processus complexe impliquant souvent les facteurs de l'hôte, du pathogène et de l'environnement. Au cours de ces dernières années, une augmentation de l'incidence des résistances aux antibiotiques des germes responsables d'infection urinaire a été constatée. L'irruption des entérobactéries sécrétrices de Bêta-Lactamase à Spectre Étendu (BLSE) est de plus en plus prévalente (4). En Afrique de l'Ouest comme à travers le monde, la résistance aux antibiotiques concerne principalement les bactéries produisant des BLSE (5). $\mathrm{Au}$ sein de la population hospitalière, la prévalence du portage de BLSE était de 10,3\% au Nigeria (6), de $21,4 \%$ dans un hôpital au Mali (7) et de 31,0\% chez les enfants hospitalisés pour malnutrition au Niger (8). Au cours d'une étude dans un orphelinat au Mali, les auteurs ont trouvé une prévalence de portage de BLSE qui variait de $63,0 \%$ chez les membres du personnel à 100,0\% chez les enfants (9).L'absence de données sur ce sujet dans notre hôpital était une limite à la connaissance de la situation épidémiologique locale et de l'antibiorésistance des entérobactéries et ne permettait pas de guider les choix des prescripteurs.L'objectif de ce travail était de décrire le profil de résistance des entérobactéries isolées dans les Examens Cyto-Bactériologiques des Urines (ECBU) réalisés à l'Hôpital du Mali de 2011 à 2015.

\section{Matériels et Méthodes}

\section{Type d'étude}

C'était une étude descriptive rétrospective réalisée au laboratoire d'analyses de biologie médicale de l'Hôpital du Mali de Bamako, du 1er septembre 2011 au 31 décembre 2015. L'ensemble des ECBU réalisés ont été inclus. Tous les ECBU positifs à entérobactéries des patients externes et hospitalisés ont été analysés, que ce soit une infection urinaire ou une colonisation urinaire. Une infection urinaire était définie par l'association d'une bactériurie supérieure ou égale à $10^{5} \mathrm{UFC} / \mathrm{ml}$ et d'une leucocyturie supérieure ou égale à $10^{4} / \mathrm{ml}$. Une colonisation était définie par l'association d'une bactériurie inférieure ou égale à $10^{4} \mathrm{UFC} / \mathrm{ml}$ et d'une leucocyturie non significative, inférieure à $10^{4} / \mathrm{ml}$. Les cas d'infections polymicrobiennes ont été exclus.

\section{Description de la réalisation de l'ECBU et de l'antibiogramme}

Chaque échantillon a fait l'objet d'un ECBU de routine comprenant un examen macroscopique pour apprécier l'aspect de l'urine et un ensemencement.

- État frais et cytologie :

L'analyse à l'état frais a permis le comptage des leucocytes et des hématies à l'aide d'un analyseur d'urines (Mindray UA-66) et la recherche de cristaux, de cellules épithéliales et de parasites au microscope optique.

- Culture bactérienne :

Un milieu chromogène Uriselect 4 a été utilisé pour la culture bactérienne avec la technique d'ensemencement suivante :

$\checkmark \quad$ Utiliser une oese calibrée de $10 \mu \mathrm{l}$;

$\checkmark$ Tenir l'oese verticalement et l'immerger dans l'urine ;

$\checkmark$ Décharger l'oese en réalisant une strie sur un rayon de la boîte ;

$\checkmark$ A partir du haut du dépôt, et sans recharger l'oese, pratiquer des stries serrées sur toute la surface de la gélose, perpendiculairement au rayon tracé au début de l'ensemencement ;

$\checkmark$ Incuber les boites à $37^{\circ} \mathrm{C}$ entre 18 et 24 heures.

- Identification :

Elle a été faite en référence à l'abaque d'identification du milieu Uriselect4, complété au besoin par des tests complémentaires simples (Uréeindole ou coloration de Gram), par une galerie biochimique classique en tubes (Kligler-Hajna, citrate de Simmons, Mannitol-Mobilité ou uréeindole) ou par une galerie API 20 E ou API 20 NE de BioMérieux.

- Test de sensibilité aux antibiotiques :

L'étude de la sensibilité aux antibiotiques a été déterminée par la méthode standard de diffusion en milieu gélosé de Mueller-Hinton (disques BioRad) selon Kirby-Bauer et interprétée selon les recommandations du Comité Antibiogramme de la Société Française de Microbiologie (CA-SFM). Le choix des disques d'antibiotique a été fait en tenant compte des phénotypes de résistance des 
entérobactéries aux bêta-lactamines (groupe I, II et III). Les disques suivants ont été utilisés : Amoxicilline, association Amoxicilline + Acide clavulanique (AMC), Céfalotine (CEF), Céfoxitine (FOX), Ceftriaxone (CRO) et Imipénème (IMP). Les résultats ont été exprimés en souches Sensibles $(\mathrm{S})$, Intermédiaires (I) et Résistantes (R). Pour l'analyse, les souches catégorisées intermédiaires ont été considérées comme résistantes. La recherche de BLSE était réalisée par la méthode de la synergie entre deux disques de Céphalosporine de 3ème génération (FOX ou CRO) et d'AMC, distants de 30 $\mathrm{mm}$. La présence d'un aspect synergique en " bouchon de champagne» confirmait la présence d'une BLSE.

\section{Recueil de données}

Les données ont été collectées à partir des registres du laboratoire de bactériologie. Un formulaire de recueil de données a été élaboré avec les variables suivantes :

- Socio-démographiques : âge, sexe et statut hospitalisé ou non ;

- Bactériologiques : nom de la bactérie isolée, bactériurie, leucocyturie et sensibilité aux antibiotiques.

\section{Saisie et analyse des données}

Les données ont été saisies et analysées avec les logiciels Excel de Microsoft et Epi info version 7.

\section{Résultats}

\section{Nombre d'ECBU réalisés et nombre d'ECBU positifs}

Sur la période de l'étude, 4578 ECBU ont été réalisés au laboratoire de bactériologie de l'Hôpital du Mali. Nous avons constaté une augmentation constante du nombre de demandes d'ECBU, avec 137 ECBU en 2011 et 1612 ECBU en 2015 (Figure 1).

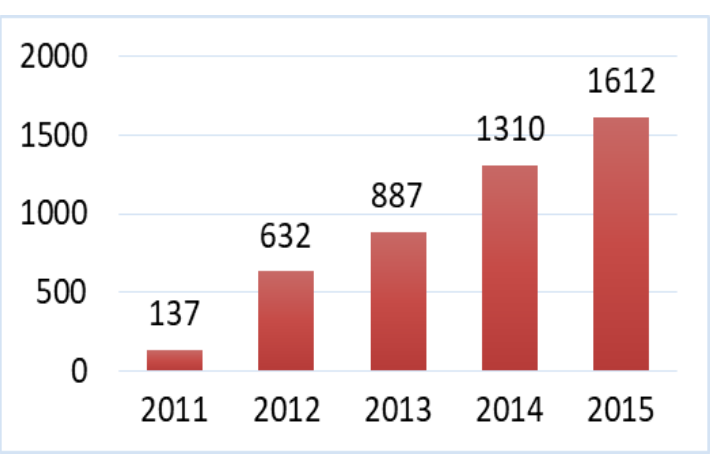

Figure 1 : Évolution du nombre d'Examens CytoBactériologiques Urinaires réalisés au laboratoire de bactériologie de l'Hôpital du Mali, de Septembre 2011 à Décembre 2015.
Les ECBU représentaient 55,2\% des examens de bactériologie prescrits (4578 sur 8298 examens bactériologiques). Parmi ces 4578 ECBU, 849 (18,5\%) étaient positifs (Figure 2).

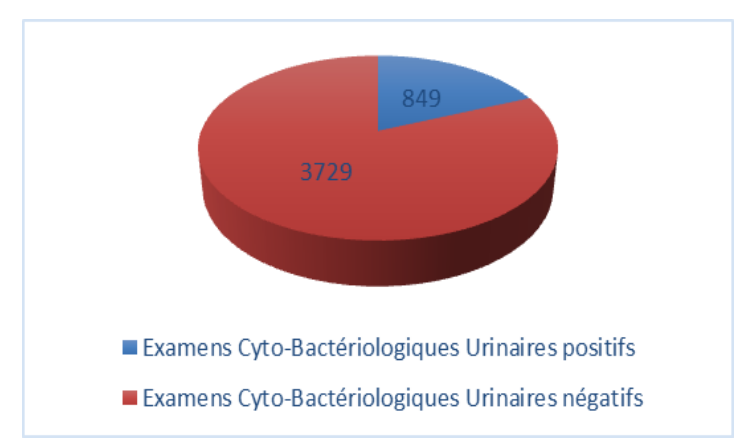

Figure 2 : Résultats des Examens CytoBactériologiques Urinaires.

\section{Caractères socio-démographiques des patients ayant réalisé un ECBU}

Parmi les 4578 patients ayant réalisé un ECBU, il y avait 2360 femmes $(51,6 \%)$ et 2218 hommes $(48,4 \%)$. Parmi les 849 patients ayant un ECBU positif, il y avait 484 femmes $(57,0 \%)$ et 365 hommes $(43,0 \%)$, soit un sexe ratio de 0,8 .

L'âge moyen des patients présentant un ECBU positif était de 36 ans, avec des âges extrêmes compris entre 1 mois et 90 ans et une médiane entre 41 et 50 ans. Les taux d'ECBU positifs par rapport aux ECBU réalisés étaient compris entre 18,0 et $22,0 \%$ chez les patients de 0 à 60 ans. Le taux d'ECBU positifs par rapport aux ECBU réalisés était le plus bas chez les patients de 61 à 80 ans, avec 9,6\%, et le plus élevé chez les patients de plus de 81 ans, avec $34,8 \%$ (Figure 3).

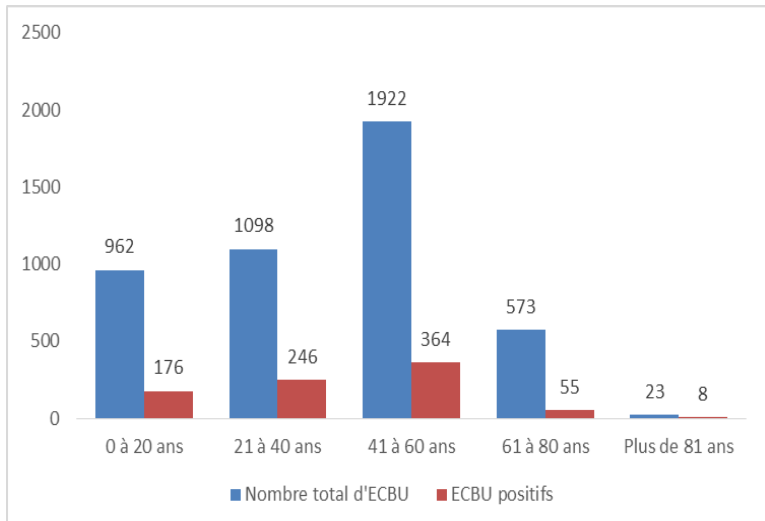

Figure 3: Résultats des Examens CytoBactériologiques Urinaires en fonction des tranches d'âges 


\section{Répartition des ECBU en fonction de la provenance du patient}

Les patients hospitalisés à l'Hôpital du Mali avaient bénéficié de 2884 ECBU (63,0\% de l'ensemble des ECBU), dont 539 ECBU positifs (63,5\% des ECBU positifs) et les patients consultants en externe avaient bénéficié de 1694 ECBU (37,0\%), dont 310 ECBU positifs $(36,5 \%$ des cas positifs).Les services où étaient retrouvés les nombres les plus importants d'ECBU positifs étaient le service de médecine avec 426 cas $(50,2 \%)$ et le service de chirurgie avec 298 cas $(35,1 \%)$ (Figure 4).

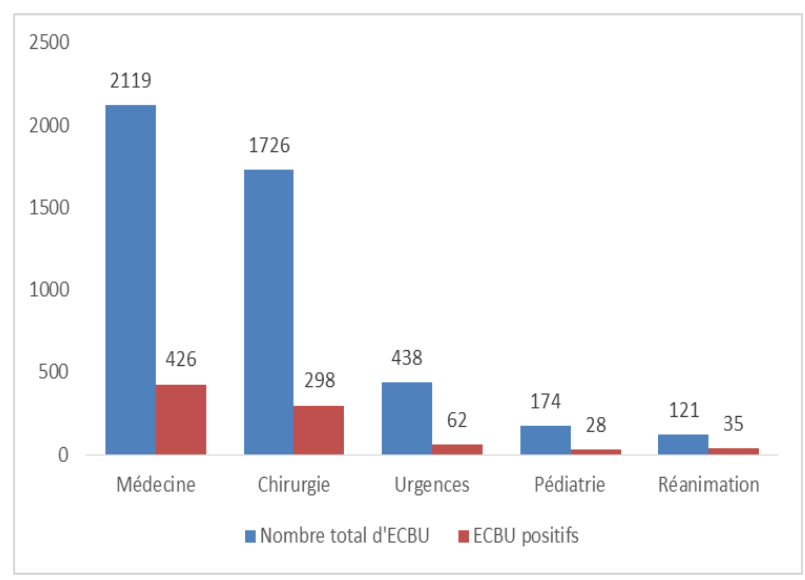

Figure 4 : Résultats des Examens CytoBactériologiques Urinaires en fonction des services des patients

Les taux d'ECBU positifs par rapport aux ECBU réalisés étaient de $14,2 \%$ dans le service d'urgences, de $16,1 \%$ dans le service de pédiatrie, de $17,3 \%$ dans le service de chirurgie, de $20,1 \%$ dans le service de médecine et de $28,9 \%$ dans le service de réanimation (Figure 4).

\section{Description bactériologique}

Une infection urinaire a été retrouvée dans $96,8 \%$ des cas $(n=822)$ et une colonisation a été retrouvée dans $3,2 \%$ des cas $(n=27)$.Les bacilles à Gram négatif étaient les bactéries les plus fréquentes, avec 651 souches identifiées (76,7\% des 849 ECBU positifs) (Figure 5). Parmi ces 651 souches de bacilles à Gram négatif, on retrouvait 569 souches : Escherichia coli était la souche la plus fréquemment isolée, avec 364 cas $(42,9 \%$ des ECBU positifs et $44,7 \%$ des infections urinaires), suivie de Klebsiella pneumoniae, avec 138 cas (16,3\% des ECBU positifs et $17,0 \%$ des infections urinaires). On ne trouvait pas de différence statistiquement significative en ce qui concernait la répartition des bactéries selon le sexe : Escherichia coli était la bactérie la plus fréquente pour les deux sexes avec 236 cas chez les femmes
$(48,8 \%)$ et 128 cas chez les hommes $(35,1 \%)$.Des levures du genre Candida étaient retrouvées dans 50 cas $(5,9 \%)$.

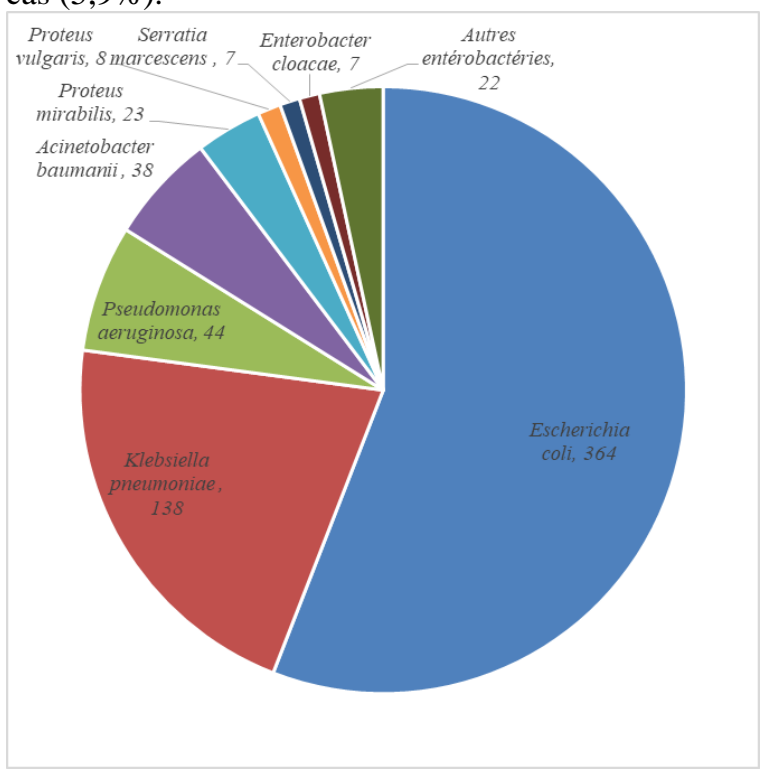

Figure 5 : Répartition des bacilles à Gram négatif identifiées.

Les autres entérobactéries représentaient toutes chacune moins de $1 \%$ des Examens Cyto-Bactériologiques Urinaires positifs : Enterobacter aerogenes, Citrobacter diversus, Klebsiella oxytocyca, Citrobacter koseri, Morganella morganii, Alcagenes dispar et Providencia sp.

\section{Analyse du profil de résistance}

Concernant la résistance aux antibiotiques, 472 des 569 souches d'entérobactéries identifiées $(83,0 \%)$ avaient une résistance acquise à l'association Amoxicilline + Acide clavulanique (Tableau I). Entre 2011 et 2015, ce taux de résistance des entérobactéries a fluctué de $71,2 \%$ à $96,6 \%$. L'ensemble des souches résistantes à l'association Amoxicilline + Acide clavulanique $(n=472)$ étaient sensibles à l'Imipénème (Tableau 1). Les entérobactéries productrices de BLSE représentaient $12,5 \%$ de l'ensemble des entérobactéries, avec respectivement 14,3\% des Escherichia coli, 12,3\% des Klebsiella pneumoniae, 8,3\% des Enterobacter aerogenes et $4,3 \%$ des Proteus mirabilis (Tableau II). Les autres phénotypes de résistances des entérobactéries étaient: 472 productrices de bêtalactamase $(83,0 \%), \quad 11$ productrices de céphalosporinase de haut niveau $(1,9 \%)$ et 0 productrice de carbapénémase $(0,0 \%)$. Plusieurs phénotypes de résistance pouvaient se cumuler chez la même entérobactérie. Seulement 61 souches étaient sauvages $(10,7 \%)$. 


\section{Discussion}

Dans cette étude réalisée sur plus de 4000 échantillons d'urines, le taux d'ECBU positifs était de $18,5 \%$. Les entérobactéries représentaient $76,7 \%$ des bactéries isolées. Des taux légèrement supérieurs (85,0\%) ont été rapportés dans la littérature [19]. La répartition par genre retrouvait une prédominance féminine avec $57,0 \%$ des ECBU positifs chez les femmes (484 sur 849 ECBU positifs) et $43,0 \%$ chez les hommes (365 sur 849 ECBU positifs). Ce même constat a été fait par d'autres auteurs [21]. Une fréquence élevée d'ECBU positifs a été retrouvée chez les patients hospitalisées $(63,5 \%)$, ce qui est similaire aux résultats de Soula et al. qui avaient trouvé un résultat de 63,6\% dans une étude réalisée en 1990 à l'Hôpital du Point $\mathrm{G}$ de Bamako au Mali (10).La majorité des patients présentant un ECBU positif provenait du service de médecine $(50,2 \%)$, ce qui est différent des résultats de Soula et al. qui avaient trouvé $18.7 \%$ en médecine et $51,5 \%$ en chirurgie (10). Ce phénomène peut s'expliquer par la répartition différente des services: présence d'un service d'endocrinologie à l'Hôpital du Mali, avec survenue fréquente d'infections urinaires chez les patients atteints d'affections métaboliques, versus présence d'un service d'urologie à l'Hôpital du Point G.

Escherichia coli était l'entérobactérie la plus isolée $(44,7 \%)$, vraisemblablement à cause de sa capacité d'adhérence aux cellules, suivie par Klebsiella pneumoniae $(17,0 \%)$, ce qui est concordant avec les résultats d'une partie de la littérature $(11,12)$ mais légèrement supérieurs à ceux d'autres travaux $(2,13)$. Escherichia coli était la bactérie la plus rencontrée chez la femme $(48,8 \%)$ comme chez l'homme (35,1\%).Sur les 4 années de l'étude, plus de 71,0\% des entérobactéries du groupe 1 isolées présentaient une résistance à l'association Amoxicilline + Acide clavulanique. Ces résultats sont similaires à ceux de Kouassi-M'bengue et al. en 2008 au Sénégal, qui retrouvaient entre $66,7 \%$ à $85,7 \%$ (14), mais ils sont inférieurs à ceux de Kara Terki et al. en 2016 en Algérie, qui retrouvaient 94,0\% (15). Cette résistance acquise serait la conséquence de la pression de sélection liée à la consommation abusive de ces antibiotiques dans les pays en développement (16). Ces taux élevés de résistance à l'Amoxicilline justifient que les amino-pénicillines ne soient plus actuellement recommandées en traitement probabiliste des infections urinaires.La présente étude a mis en évidence la présence de BLSE parmi 12,5\% des entérobactéries. Ces résultats sont inférieurs à ceux trouvés dans un autre hôpital du Mali de 21,5\% (7). Cette résistance pourrait s'expliquer par une baisse de l'activité de l'inhibiteur des bêta-lactamases (Acide clavulanique), résultante d'une hyperproduction de pénicillinase, ou par l'inactivation de l'inhibiteur lui-même (15). Cela est probablement dû à la prescription souvent empirique de cette molécule particulièrement en médecine ambulatoire dans l'attente des résultats des ECBU. $\mathrm{Ce}$ résultat est légèrement inférieur à celui de Moutachakkir et al. en 2014 (17) qui avaient trouvé $17,5 \%$. Ces mêmes auteurs avaient trouvé $99,0 \%$ de souches sensibles à l'Imipenème contre 100,0\% dans cette étude. Nos résultats sont similaires à ceux rapportés par d'autres auteurs $(2,18)$.Selon la 5ème enquête démographique et de santé du Mali (EDSM $\mathrm{V}$ 2012-2013), 52,0\% de la population urbaine et $63,0 \%$ de la population rurale ont pratiqué l'automédication. L'éducation de la population, l'interdiction de vente d'antibiotiques sans ordonnance et la minimisation de l'antibiothérapie probabiliste dans les services d'hospitalisation pourraient être des solutions pour limiter la résistance aux antibiotiques.

\section{Conclusion}

L'infectiologie est une spécialité où il est primordial d'adapter la prise en charge thérapeutique d'une pathologie à l'agent infectieux en cause et à ses résistances, dans un objectif de santé publique et de limitation de l'augmentation des résistances aux traitements anti-infectieux.Cette étude nous a permis de connaître le profil de résistance aux bêtalactamines des entérobactéries identifiées dans les prélèvements urinaires de l'Hôpital du Mali. Nous avons retrouvé un taux extrêmement élevé de résistance à l'association Amoxicilline + Acide clavulanique et $12.5 \%$ de souches productrices de BLSE.Grâce à cette étude, des discussions pourront être mises en place entre les biologistes, les pharmaciens et les médecins des principaux services prescripteurs d'ECBU, pour adapter au mieux les prescriptions à l'écologie bactériologique locale et mettre en place des protocoles d'antibiothérapie.

Conflict d'intérêt : aucun 


\section{Références}

1. Bléry, M., Tasu JP, Rocher L, Rondeau Y, Miquel A. Infection urinaire aiguë non tuberculeuse de l'adulte. J Radiol. 2000 Sep; $81: 1028-36$.

2. Lo S, Ka R, Ba Diallo A, Diallo OF, Diagne R, Dia ML, Sarr AM, Sow AI. Sensibilité aux antibiotiques des Entérobactéries isolées d'urines au Centre Hospitalier Régional de Saint Louis (Sénégal) de juin 2011 à juillet 2012. Rev Afr Malgache Rech Sci Santé. 2015 Mar 8;2(2).

3. Ouedraogo AS, Pierre HJ, Bañuls AL, Ouédraogo R, Godreuil S. Émergence et diffusion de la résistance aux antibiotiques en Afrique de l'Ouest: facteurs favorisants et évaluation de la menace. Médecine Santé Trop. 2017 May;27(2):147-54.

4. WHO | Antimicrobial resistance: global report on surveillance 2014 [Internet]. [cited 2019 Nov 1]. Available from: https://www.who.int/antimicrobial-

resistance/publications/surveillancereport/en/

5. Pitout JDD, Laupland KB. Extended-spectrum beta-lactamase-producing Enterobacteriaceae: an emerging public-health concern. Lancet Infect Dis. 2008 Mar;8 (3):159-66.

6. Olowe OA, Grobbel M, Büchter B, LübkeBecker A, Fruth A, Wieler LH. Detection of bla(CTX-M-15) extended-spectrum betalactamase genes in Escherichia coli from hospital patients in Nigeria. Int $\mathbf{J}$ Antimicrob Agents. $2010 \mathrm{Feb}$;35(2):206-7.

7. Duval V, Maiga I, Maiga A, Guillard T, Brasme L, Forte D, et al. High Prevalence of CTX-MType $\beta$-Lactamases among Clinical Isolates of Enterobacteriaceae in Bamako, Mali. Antimicrob Agents Chemother. 2009 Nov;53(11):4957-8.

8. Woerther P-L, Angebault C, Jacquier H, Hugede H-C, Janssens A-C, Sayadi S, et al. Massive increase, spread, and exchange of extended spectrum $\beta$-lactamase-encoding genes among intestinal Enterobacteriaceae in hospitalized children with severe acute malnutrition in Niger. Clin Infect Dis Off Publ Infect Dis Soc Am. 2011 Oct;53 (7):677-85.

9. Tandé D, Jallot N, Bougoudogo F, Montagnon T, Gouriou S, Sizun J. Extended-Spectrum $\beta$ Lactamase-Producing Enterobacteriaceae in a
Malian Orphanage. Emerg Infect Dis. 2009 Mar;15(3):472-4.

10. Soula GH, Pichard E, Soula GG, Kodio A. Etude bactériologique des infections urinaires à Bamako: orientation pratique. Médecine Afr Noire. 1990;5:37.

11. Toudji AG, Djeri B, Karou SD, Tigossou S, Ameyapoh Y, Souza C de. Prévalence des souches d'entérobactéries productrices de bêtalactamases à spectre élargi isolées au Togo et de leur sensibilité aux antibiotiques. Int $\mathrm{J}$ Biol Chem Sci. 2017 Jan;11(3):1165-77.

12. Kone J, Bellahcen B, Awab A, El Moussaoui R, Alilou M, El Hijri A, Azzouzi A. Les entérobactéries sécrétrices de béta-Lactamases à spectre étendu en urologie à l'Hôpital Ibn Sina de Rabat. Rev Malienne D'Infectiologie Microbiol. 2016 Aug;7:2-7.

13. Sekhsokh Y, Chadli M, Hamzaoui SAE. Fréquence et sensibilité aux antibiotiques des bactéries isolées dans les urines. Médecine Mal Infect. 2008 Jun 11;38(6):324-7.

14. Kouassi-M'bengue A, Folquet-Amorissani M, Nassirou F, N.Guessennd-Kouadio1 A. KacouN'Douba, Houenou Y, Dosso M. Les infections urinaires néonatales à Abidjan: problématique de la résistance bactérienne. Mali Méd. 2008;23(1):34.

15. Kara Terki I, Hassaine H, Bellifa S, M'hamedi I, Lachachi M. Infection urinaire nosocomiale: Etude prospective dans une unité de réanimation médicale à l'ouest Algérien. Rev Microbiol Ind Sanit Environnementale. 2012;6(1):118-30.

16. Gonsu Kamga H, Nzengang R, Toukam M, Sando Z, Shiro S. Phénotypes de résistance des souches d'Escherichia coli responsables des infections urinaires communautaires dans la ville de Yaoundé (Cameroun). Afr J Pathol Microbiol. 2014 Jan;3:1-4.

17. Moutachakkir M, Chinbo M, Elkhoudri N, Soraa $\mathrm{N}$. La résistance aux antibiotiques chez les entérobactéries uropathogènes en milieu pédiatrique au CHU de Marrakech. J Pédiatrie Puériculture. 2015;28(1):16-22.

18. Hamze M, Dabboussi F, Izard D. Sensibilité des entérobactéries aux antibiotiques: étude sur quatre ans (1998-2001) dans le nord du Liban. Cah D'études Rech Francoph. 2003;13(2):10712. 
$\underline{\text { Tableau I }}$ : Profil de sensibilité et de résistance des entérobactéries aux antibiotiques testés

\begin{tabular}{|c|c|c|c|c|c|c|c|c|c|c|c|c|c|c|}
\hline \multirow{3}{*}{ Entérobactéries } & \multirow{3}{*}{ Germes } & \multirow{3}{*}{ Effectif } & \multicolumn{12}{|c|}{ Résistance acquise aux bêta-lactamines } \\
\hline & & & \multicolumn{2}{|c|}{ AMX } & \multicolumn{2}{|c|}{ AMC } & \multicolumn{2}{|c|}{ CEF } & \multicolumn{2}{|c|}{ FOX } & \multicolumn{2}{|c|}{ CRO } & \multicolumn{2}{|c|}{ IPM } \\
\hline & & & $\mathbf{S}$ & $\mathbf{I} / \mathbf{R}$ & $\mathbf{S}$ & $\mathbf{I} / \mathbf{R}$ & $\mathbf{S}$ & $\mathbf{I} / \mathbf{R}$ & $\mathbf{S}$ & $\mathbf{I} / \mathbf{R}$ & $\mathbf{S}$ & $\mathbf{I} / \mathbf{R}$ & $\mathbf{S}$ & $\mathbf{I} / \mathbf{R}$ \\
\hline \multirow[b]{2}{*}{ Groupe I } & E. coli & 364 & 28 & 336 & 62 & 302 & 357 & 07 & 355 & 09 & 323 & 41 & 364 & 00 \\
\hline & $\begin{array}{c}P . \\
\text { mirabilis } \\
\end{array}$ & 23 & 03 & 20 & 04 & 19 & 21 & 02 & 23 & 00 & 23 & 00 & 23 & 00 \\
\hline \multirow[t]{3}{*}{ Groupe II } & $\begin{array}{c}K . \\
\text { pneumonia } \\
e\end{array}$ & 138 & - & - & 23 & 115 & 134 & 04 & 136 & 02 & 133 & 05 & 138 & 00 \\
\hline & K.oxytoca & 05 & - & - & 01 & 04 & 05 & 00 & 05 & 00 & 05 & 00 & 05 & 00 \\
\hline & C.diversus & 04 & - & - & 01 & 03 & 04 & 00 & 04 & 00 & 04 & 00 & 04 & 00 \\
\hline \multirow{8}{*}{ Groupe III } & $\begin{array}{c}E . \\
\text { aerogenes }\end{array}$ & 12 & - & - & - & - & - & - & - & - & 11 & 01 & 12 & 00 \\
\hline & P.vulgaris & 08 & - & - & - & - & 08 & 00 & 08 & 00 & 08 & 00 & 08 & 00 \\
\hline & $\begin{array}{c}S . \\
\text { marcescen } \\
S \\
\end{array}$ & 07 & - & - & - & - & 07 & 00 & 07 & 00 & 07 & 00 & 07 & 00 \\
\hline & E. cloacae & 03 & - & - & - & - & - & - & - & - & 03 & 00 & 03 & 00 \\
\hline & C. koseri & 02 & - & - & 01 & 01 & 02 & 00 & 02 & 00 & 02 & 00 & 02 & 00 \\
\hline & $\begin{array}{c}M . \\
\text { morganii }\end{array}$ & 01 & - & - & - & - & - & - & 01 & 00 & 01 & 00 & 01 & 00 \\
\hline & $\begin{array}{l}\text { Providenci } \\
\quad \text { a sp }\end{array}$ & 01 & - & - & - & - & 01 & 00 & 01 & 00 & 01 & 00 & 01 & 00 \\
\hline & A. dispar & 01 & 00 & 01 & 00 & 01 & 01 & 00 & 01 & 00 & 01 & 00 & 01 & 00 \\
\hline
\end{tabular}

AMX : Amoxicilline, AMC : Amoxicilline + Acide clavunique, CEF : Céfalotine, FOX : Céfoxitine, CRO Ceftriaxone et IPM : Imipénème. $S$ : Sensible et I/R : Intermédiaire / Résistant. - : résistance naturelle. 
Tableau II : Les phénotypes de résistance des entérobactéries testées.

\begin{tabular}{|c|c|c|c|c|c|c|}
\hline & & \multicolumn{5}{|c|}{ Phénotype } \\
\cline { 3 - 7 } Entérobactéries & Effectif & Sauvage & $\begin{array}{c}\text { Pénicillinase } \\
\text { (basée sur } \\
\text { Amoxicilline) }\end{array}$ & BLSE & $\begin{array}{c}\text { Céphalosporinase } \\
\text { de haut niveau } \\
\text { (basée sur } \\
\text { Céfoxitine) }\end{array}$ & Carbapénémase \\
\hline E. coli & 364 & 28 & 302 & 52 & 9 & 0 \\
\hline K. pneumoniae & 138 & 23 & 115 & 17 & 2 & 0 \\
\hline P. mirabilis & 23 & 3 & 19 & 1 & 0 & 0 \\
\hline E. aerogenes & 12 & 1 & 10 & 1 & 0 & 0 \\
\hline P.vulgaris & 8 & 1 & 7 & 0 & 0 & 0 \\
\hline S. marcescens & 7 & 1 & 6 & 0 & 0 & 0 \\
\hline K.oxytocyca & 5 & 1 & 4 & 0 & 0 & 0 \\
\hline C.diversus & 4 & 1 & 3 & 0 & 0 & 0 \\
\hline E. cloaceae & 3 & 1 & 2 & 0 & 0 & 0 \\
\hline C.koseri & 2 & 1 & 1 & 0 & 0 & 0 \\
\hline M. morganii & 1 & 0 & 1 & 0 & 0 & 0 \\
\hline A.dispar & 1 & 0 & 1 & 0 & 0 & 0 \\
\hline Providencia $s p$ & 1 & 0 & 1 & 0 & 0 & 11 \\
\hline Total & 569 & 61 & 472 & 71 & & 0 \\
\hline
\end{tabular}

BLSE : Bêta-lactamase à spectre élargi ou étendu. 